\title{
Measurement and Control System Based on Wireless Senor Network for Granary Song Jian ${ }^{1,2}$, Wang Kai ${ }^{2}$, Zhang Xiaochen ${ }^{2}$ \\ ${ }^{1}$ College of machinery, Weifang University, Weifang 261061, China; ${ }^{2}$ Colege of Mechanical Engineering, Chang zhou University, Changzhou ,213000, China
}

Keywords: Granary; CC2530; Zigbee, wireless senor network; Measurement and control system

\begin{abstract}
A wireless measurement and control system for granary is developed for the sake of overcoming the shortcoming of the wired measurement and control system such as complex wiring and low anti-interference capacity. In this system, Zigbee technology is applied with Zigbee protocol stack development platform by TI, and wireless senor network is used to collect and control the temperature and the humidity. It is composed of the upper PC, central control node based on CC2530, sensor nodes, sensor modules and the executive device. The wireless sensor node is programmed by $\mathrm{C}$ language in IAR Embedded Workbench for MCS-51 Evaluation environment. The upper PC control system software is developed based on Visual C++ 6.0 platform. It is shown by experiments that data transmission in the system is accurate and reliable and the error of the temperature and humidity is below $2 \%$, meeting the functional requirements for the granary measurement and control system.
\end{abstract}

\section{Introduction}

In recent years, with the sustained and stable agriculture production increase, Chinese annual grain output and perennial grain stocks have been ranking first in the world. It is vital to accurately and timely master the changes of various physical factors in order to reduce loses in the process of grain storage and safeguard the grain quality and quantity ${ }^{[1,2]}$. Currently, primitive storage manner is still used in the majority of grain stocks in our country, in which point-to-point measurement by workers is needed with a low efficiency and poor accuracy. In addition, there exist such shortcomings as complex wiring, low anti-interference capacity and difficult maintenance, which restrict its popularization and application ${ }^{[3]}$. Wireless Sensor Network (WSN) is an intelligent measurement and control network which is constituted through self-organizing of a large number of tiny lowpriced sensor nodes with the ability of communicating, sensing and computing inside or nearby the monitoring areas ${ }^{[4,5]}$.

Since the late 1990s, some scientists in Europe and America have carried on their studies on the wireless measurement and control system, in which Zigbee technology with low power consumption and high reliability is applied, and they have made some experimental achievements $[6,7,8]$. However, there are still many problems to be solved urgently in the development and appliance of the Zigbee-based measurement and control system ${ }^{[9,10]}$. In order to effectively control the granary environment and improve the production and management level, a granary measurement and control system based on Zigbee wireless sensor network is developed in this paper, with radio frequency chip CC2530 as its core, coordinating with temperature sensor, humidity sensor and the executive device, thus implementing the task of monitoring and controlling the temperature and humidity in the granary. 


\section{The System Project Design}

\section{A. Working Principle of ZigBee Technology.}

ZigBee is a wireless communication technology with such characteristics as low rate, close range, low power consumption, low complexity, low-cost, reliable communication, and high network capacity [7, 8]. The physical layer (PHY) and the media access control (MAC) of ZigBee protocols conform to IEEE802. 15.4 standards, the network layer is formulated by the ZigBee Alliance, and the application layer is permitted to develop in line with the users' application demands. ZigBee operation frequency channels fall into three bands: $868 \mathrm{MHz}, 915 \mathrm{MHz}$ and 2. $4 \mathrm{GHz}$. In the 2.4 $\mathrm{GHz}$ band there are 16 ZigBee channels, which are of global standards, free of charge, nonapplication, with the data transmission rate of $250 \mathrm{Kbps}$. The collision-proof multi-carrier channel access mode is adopted in communication so as to avoid the collision among radio carriers; the encryption algorithm with a key length of 128 bits is used to encrypt data in order to safeguard the security and secrecy of communication information.

\section{B. Network Topology Structure}

Appropriate network topology must be chosen in order to guarantee the reliable service of the system. ZigBee standard supports three main self-organization wireless networks, namely, star topology, net topology, and clusters topology. There is only one FFD node in the star network topology, with each RfD node sharing channel and only one FFD node communicating with RfD node in a certain time. Although the maximum RFD node attachable is not too large, the node communication range can satisfy the need for the granary measurement and control system as the RFD nodes in the granary wireless network have simple function and don't need routing function. The star network topology is chosen when network capacity and nodes power consumption and stability are all considered.

\section{Overall Project Design of the System}

According to the features and demands of system, a wireless measurement and control system designed which has a central control node and five sensor nodes. As Fig.1 shows, the measurement and control system is composed of the upper PC, CC2530-based center control node, sensor nodes, sensor modules and the executive device.

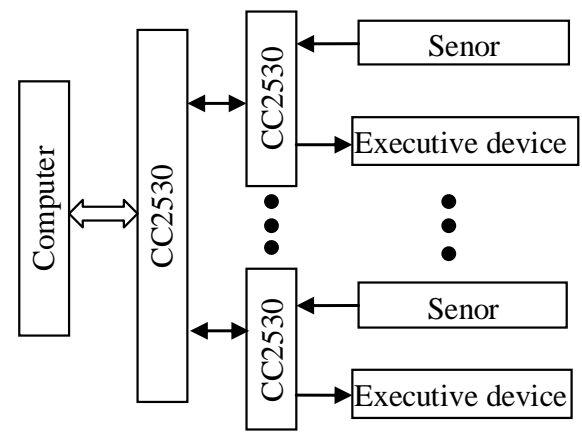

Fig. 1 Schematic diagram of the control system

The upper PC, connected with the network center node through RS232 serial port, receives data from Zigbee network and sends control instructions, monitors the greenhouse running state, displays real-time all kinds of such environmental message as temperature, humidity, also is able to complete the functions of data management and statistical analysis of the historical data. The central control node is the wireless network center, in charge of the network establishment and management. The wireless data collecting module is used to receive data from the sensors and transmit them to the central control node, meanwhile to receive the control instructions for the actuators from the upper control PC transmitted through the central control node. 


\section{System Hardware Design}

\section{A. Central Control Node}

CC2530 is a true system-on-chip (SoC) solution for IEEE 802.15.4, Zigbee and RF4CE applications. It enables robust network nodes to be built with very low total bill-of-material costs. The CC2530 combines the excellent performance of a leading RF transceiver with an industrystandard enhanced $8051 \mathrm{MCU}$, in-system programmable flash memory, 8-KB RAM, and many other powerful features. The $\mathrm{CC} 2530$ comes in four different flash versions: CC2530F32/64/128/256, with 32/64/128/256 KB of flash memory, respectively.

CC2530 has various operating modes, making it highly suited for systems where ultra low power consumption is required. Short transition times between operating modes further ensure low energy consumption. Combined with the industry-leading and golden-unit-status Zigbee protocol stack (ZStack $^{\mathrm{TM}}$ ) from Texas Instruments, the CC2530F256 provides a robust and complete Zigbee solution. Combined with the golden-unit-status Remo TI stack from Texas Instruments, the CC2530F64 and higher provide a robust and complete Zigbee RF4CE remote-control solution.

\section{B. Sensor Nodes}

The wireless sensor network nodes are made up of sensor module, processor module, wireless communication module and power module. The processor module and wireless communication module are integrated on CC2530 chip, which greatly simplifies the radio-frequency circuit design. The temperature sensor DS18B20 and humidity sensor HM1500 and infrared sensor GH-718 are used in the sensor module while the fan motor is driven by VVVF tailored for fan in the executive device. The node hardware functional block diagram is shown as in Fig.2.

The single-wire digital temperature sensor DS18B20 produced by American DALLAS semiconductor company is employed in the temperature acquisition module, which is in 3-pined TO-92 small volume seal form. The temperature measurement range is from -55 to $+125^{\circ} \mathrm{C}$, programmable for 9-12 bits conversion accuracy and temperature measurement resolution reaches $0.0625{ }^{\circ} \mathrm{C}$. The sign-extended 16 bits digital value serial output is used for the measured temperature. It requires only one port wire for DS18B20 CPU to communicate with numerous DS18B20, which occupies less microprocessor port so that it can save plenty of leads and logic circuits.

HM1500 is specialized for conditions needing accurate and reliable humidity detection. It has high measurement accuracy, stable and reliable measuring result and a long service life. It converts humidity into analog electrical signal which is through output resistance then converted into voltage signal that is converted into digital value via $\mathrm{AD}$ converter. The humidity sensor is used for measuring air humidity inside and outside of the granary, the result of which is transmitted through CC2530 chip to the central control computer which controls the relay according to the measurement result for whether or not to turn on the ventilation fan.

GH-718 infrared sensor for human body is adopted for anti-theft sensor with the following features: no need for emitter of infrared ray or electromagnetic wave; high sensitivity and wide control range; good concealment and moveable installment suitable for anti-theft alarm device.

The system regulation and control executive device is the ventilation and humidity-reducing device driven by the electric motor which is controlled by the frequency converter. The ventilation fan works in the long-term continuous state, which belongs to continuous constant load. So there will be no overload in normal conditions. AC frequency conversion speed regulator specialized for fan is chosen in view of the above two points.

\section{System Software}

\section{A. Upper PC Software}

The main function of upper PC software is to communicate with the central control node located in the monitoring room through RS-232 serial port so as to receive sensor node message. Moreover, 
upper PC software should achieve such functions as human-computer interaction, network management, sensor message sink, data processing and analysis, and database management.

Mscomn, the communication control package of Visual $\mathrm{C}++6.0$, can provide full functions of serial communication, able to read in or write data from or to serial ports. It is simple and convenient to compile and debug program with a fast development rate, thus conveniently realizing asynchronous serial communication between users and the application program. Mscomn control package provides data transmitting and receiving function for the application system through the serial port, which is very simple. Moreover, it realizes good human-computer interface and meets the basic demands of wireless sensor network. Fig. 2 is the schematic diagram of upper monitor control PC.

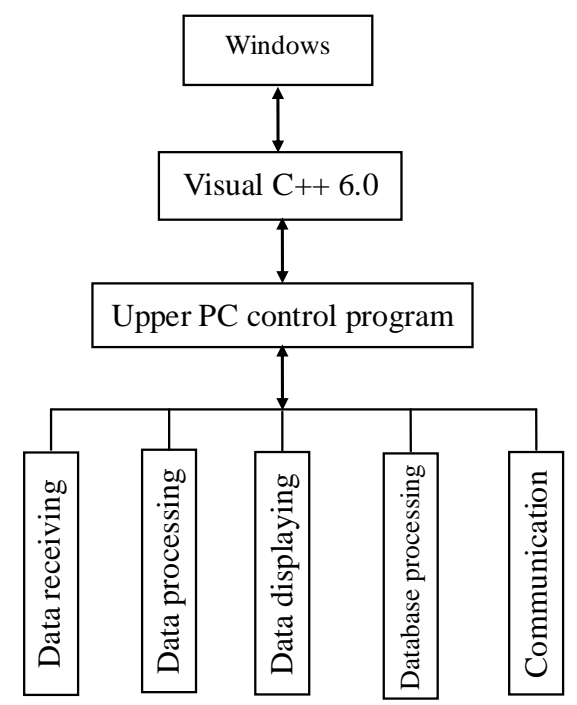

Fig. 2 Schematic diagram of upper monitor control PC

\section{B. Sensor Node Program}

Sensor node is mainly in charge of collecting the temperature and the humidity information, transmitting data, receiving and conducting the control commands. When the node initialization is started, it actively scans valid network channels and searches nearby the appropriate coordinator, and applies for access to the network. It formally accesses network after it wins approval, then it begins the operation of collecting and transmitting data and receiving and conducting the commands. It employs the duty cycle operation mode, while it is in sleeping mode when not working in order to reduce power consumption for energy-saving purpose. In IAR Embedded Workbench for MCS-51 Evaluation, C language is used to develop sensor node software, conducting program editing, compiling, linking and debugging.

\section{Experiment and Analysis}

In order to guarantee the measurement and control system to work normally and reliably, experiment is carried out to test the validity of communication pact, communication distance and rate index, and experimentally analyze and rate the sensors' accuracy. The test system is a star network topology consisting of one central control node and five sensor nodes.

Through the tests, the system can transmit data accurately and reliably with the data loss rate of 0 . After numerical values of each sensor node which are read at eight different times are analyzed and rated, it is found that the data measured is very close to the actual numerical value with an error rate within $2 \%$ although there are errors among data from different nodes. Furthermore, it has the features of simple wiring, stable and reliable performance and high accuracy in measurement and control. 


\section{Conclusion}

The wireless measurement and control system for granary is put forward for the sake of controlling effectively the environmental targets in the granary. In the system, Zigbee technology is applies to the granary measurement and control system, and wireless senor network is used to collect and control the temperature and the humidity. The wireless senor network is designed, and the software and hardware development of the sensor nodes are accomplished. It is illustrated by running experiments that the measurement and control system based on Zigbee wireless sensor network has such features as high reliability, strong anti-interference, low power consumption and low costs and can meet the functional requirements for the granary measurement and control system.

\section{Acknowledgment}

This work is supported by Shandong Provincial Natural Science Foundation, china (No.Y2008G32) and Shandong Provincial universities Scientific Research Project (No.J09LG53).

\section{References}

[1] Y.J. Zhao, J.L. Ma, Y. Qing, Research on Monitoring System for Granary Based on LabVIEW, J. Journal of Agricultural Mechanization Research, 30(2009),111-113

[2] Y.X. Liu, Design Granary Environment Measure and Control System Based on ZigBee Wireless Sensor Network, J. Computer \& DigitalEngineering, 36(2010) 74-77

[3] L. Li, Distributed Sensors Network of Granary Based on Zigbee, J. Network and Information Technology, 31(2012) 63-65

[4] S.F. Du, Y.X. Li, C.W. Ma, Current situation on greenhouse environment control system modes in China, J. Agricultural Engineering, 30(2011) 7-12

[5] S.G. Liu, J.W. Ma, R.Z. Shi, Research and development of measuring and controlling system for agricultural facilities, J. Agricultural Engineering, 27(2011) 246-249

[6] N. Wang, N.Q. Zhang, M.H. Wang, Wireless sensors in agriculture and food industry-Recent development and future perspective, J. Computers and Electronics in Agric, 50(2006)1-14

[7] R.X. Jiang, K.Y. Lin, J.H.Wu, Study \& Design on Monitoring and Controllig System for Greenhouse Group Based on ZigBee, J. Journal of Shanghai Jiaotong University (agricultural science edition), 26(2008) 440-444

[8] F. Cali, M. Conti, Gregori E. IEEE 802.11 protocol: Design and performance evaluation of an adaptive backoff mechanism. IEEE Journal on Selected Areas in Communications, 28(2010) 17741786

[9] R.C. Wang. Wireless Sensor Network Technology and Application, People's Posts and Telecommunications Press, Beijing, 2012

[10] F.I. AkyilizI, weiliansu, E. Cayirei. Asurvey on Sensor networks. IEEE Communications Magazine, 40(2000) 102-114 